\title{
The Line-broken Analysis of the Aluminum Conductor Composite Core
}

\author{
Chufan Xue ${ }^{1}$, Dehong Wang ${ }^{2}$, Yong Tang ${ }^{1}$, Guorong Chen ${ }^{1}$, Yongchang Lin ${ }^{1}$, Junfeng Bai, Yanzhong Ju² ${ }^{2}$ \\ ${ }^{1}$ Qingyuan Power Supply Bureau of Guangdong Power Grid Corporation, Qingyuan 511500, China \\ ${ }^{2}$ Country School of Civil Engineering and Architecture, Northeast Electric Power University, Jilin 132012, China
}

\begin{abstract}
Aluminum conductor composite core has many technical advantages such as high tensile strength, light weight, low sag, large current carrying capacity. In order to study the influence of the broken of aluminum conductor composite core on the transmission tower, tower-line model was built by using the finite element software ANSYS/LS-DYNA, and the deformation of joints and stress of bar were analyzed. The impact load and the impact coefficient of the representative bars of the transmission tower are calculated. The results show that the impact acting on the tower is the most serious in the place of the conductor suspension point, the connection of upper and lower crank arms, the tower neck.
\end{abstract}

\section{Introduction}

The line-broken load is the tension difference between two-phase conductor after one-phase conductor loses its tensile stress at a short time, and the line-broken oscillation is one of the vertically unbalanced loads acting on the transmission tower. When the conductor of a certain span is broken, the unbalanced tensile stress due to cable rupture will have a great influence on the transmission tower that connects with the conductor, and the distribution of the deformation and the stress of the tower that mainly bears vertical load has great changes, which may cause a serious threat to the transmission tower even the whole line. The unbalanced tensile stress in the direction along the line is an important part of design loads of the transmission tower [1]. Consequently, the research for the impact load due to cable rupture have great significance for the structural design of the transmission tower. Producing test and the numerical simulation of the transmission tower-line system model, John Mozer [2] obtained the static and dynamic responses of the transmission tower along the line, and by comparing with the analytical method, they predicted the operating load under the normal condition and the impact load at dynamic peak value along the line. John [3] analyzed the dynamic impact response of the transmission tower-line system when one or more conductors suddenly broken, and the measured impact coefficient could reach up to 4.5. Thomas [4] thought that the time history of the load should be considered in the research for the impact load of the transmission tower-line system due to cable rupture, and they proposed the time-history calculating method for the cable rupture based on the different model and parameters of the conductor. By summarizing the line-broken projects from the electricity research department, Mozer [5] proposed a balanced solution for the line-broken analysis of the transmission line based on the nonlinear stiffness analysis of the transmission tower-line system, and they ultimately gave the specific steps in the analysis of the unbalanced tensile stress due to cable rupture.

Ghyslaine [6] conducted a nonlinear dynamic analysis of the system with ANYDA program, and they separately used the small-size model under four ideal states to made the dynamic analysis for the transmission tower-line system. Xia[7] used the finite element software ANSYS/LS-DYNA to build the conductors as well as the ground wires models, replaced the tower-spring model under the action of conductors and ground wires on the tower with an equivalent spring model, and they simulated the dynamic response of the transmission tower under the condition that the conductors or the ground wires were broken. The tower-line model not only considers the action of conductors and ground wires on the tower, but also simplifies the modeling and calculation of the towerline system. However, the tower-line coupling effect is not fully considered in this model, and there may exist some deviations in the results. Bao [8] used the ABAQUS software to simulated the transient fracture and free fall of the conductor with and without consideration of the ground, and the impact load and the impact coefficient under the line-broken condition were calculated. Tan [9] analyzed the dynamic response of the long span transmission tower under the line-broken condition using the model that includes two towers and three lines, taking the Hanjiang big span project as a part of the $1000 \mathrm{kv}$ transmission line from southeast Shanxi to Nanyang to Jinmen for example. Zhou [10] calculated the line-broken effect on the tower and the unbalanced tensile stress of the conductors in the extreme weather conditions. Lu [11] built the finite element model of the transmission tower-

*Corresponding author's e-mail: juyanzhong@126.com 
line system with five spans, and analyzed static characteristics, dynamic characteristics and the linebroken problem of the whole system. Due to the shortcomings of the quasi-static method, Li [12] used the explicit dynamic finite element method to make a research for the oscillatory response after the cable rupture, and analyzed the influence on the line-broken response analysis for the difference of the initial tension and the span. Shen [13] simulated the process of the transmission line breakage by using the life-death element method, and using contact and friction to simulate the interaction between conductors and ground wires, and finally obtained the line-broken tension and response of the transmission line.

The aluminum conductor composite core has many characteristics, including self-damping, small density, low coefficient of thermal expansion, as well as high tensile strength, and it's physical property and mechanical property have remarkable difference from the steel-cored aluminum strand, so that the line-broken response of the aluminum conductor composite core in the line should have its own characteristics. At present, the research for the line-broken response of the aluminum conductor composite core is limited, so the promotion of the research is of great practical significance to improve the construction, installation, specification of such conductors.

\section{The Foundation of The Model of The Transmission Tower-line System}

\subsection{The Finite Element Model of The Transmission Tower}

This chapter chooses the 5A-ZM4 cat-head linear tower as the research object in the fascicle of $500 \mathrm{kv}$ transmission line of typical design for transmission and transformation engineering of the State Grid Corporation. The single line diagram of the tower is shown in figure 1 . When ANSYS/LS-DYNA was used to establish the finite element model of the transmission tower, the main members are simulated by using the spatial beam element BEAM161, the diagonal members and the auxiliary members are simulated by using the space bar element LINK160.

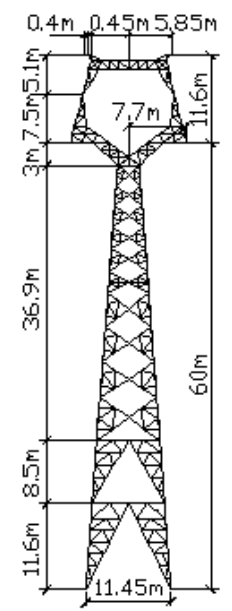

Figure 1 The single line diagram of the tower 5A-ZM4
As a kind of high flexibility component, the conductor's characteristic is that it can only bear tension but not bending moment and pressure, and we can accurately deal with it as the single suspension structure [15-16]. Based on the above, LINK167 is used to display cable element for simulation of the conductor in this paper. The LINK167 element is the same as the elastic element, and the correlation between force and deformation is directly inputted by the users. In the ANSYS/LS-DYNA program, the cable rupture is controlled by the material failure [17-18]. According to the places that the cable rupture more frequently occurs to, the location near the hanging point of the side conductor is selected as the linebroken place.

\subsection{The Line-broken Analysis}

According to the results of calculation, we can know that the impact effect at a more serious level acts on many places, including the connection of upper and lower crank arms in the tower head(node 535), the hanging point of the line-broken conductor(node 562), the tower neck, the diaphragm surface in the tower body. Then we extract the replacement-time curves of the node 535, 562 and 520(the hanging point of the ground wire). In the duration of $0 \sim 7.5 \mathrm{~s}$ after the cable rupture, the deformation of node 535 changes in the range of $0 \sim-0.412 \mathrm{~m}$, and reaches its maximum value- $0.412 \mathrm{~m}$ at $0.512 \mathrm{~s}$, the change of the node deformation gradually Slows down then. In the duration of $0 \sim 7.5 \mathrm{~s}$, node 562 have a little larger range of the node deformation than node 535 , and at $0.512 \mathrm{~s}$ the deformation reaches its maximum, $0.421 \mathrm{~m}$, the change of the node deformation gradually getting slow then, but the deformation is larger than node 535. Node 520 locates at the support of ground wires in the non-broken side, the deformation of node changes in the small range of 0.089 $0.280 \mathrm{~m}$, and at $0.813 \mathrm{~s}$ reaches its maximum, $0.280 \mathrm{~m}$.

The connection bar of upper and lower crank arms in the tower head is under the tension state due to the large line-broken tension after cable rupture. The stress change is dramatic at the first $0.4 \mathrm{~s}$ after cable rupture, and change is not significant after $1.6 \mathrm{~s}$ and tends to be steady. The stress of the bar 1066 has a small oscillation within $0 \sim 0.57 \mathrm{~s}$ after the cable rupture, and the maximum value of the compressive stress is $54.532 \mathrm{Mpa}$ in this duration. Then the stress of the bar 1066 (a bar used for hanging wire) has a fierce change that the stress increase to 77.164MPa at an extremely short time, gradually getting steady after $1.8 \mathrm{~s}$, and bar 1066 bears compression stress from beginning to end. Within $0 \sim 0.12 \mathrm{~s}$, the stress of bar 1581 (one of connection bar of tower head and tower body) rapidly reaches $20.902 \mathrm{MPa}$, and the stress value fiercely add up to $37.644 \mathrm{MPa}$ after several changes then, at $0.986 \mathrm{~s}$ reaching its maximum, $39.245 \mathrm{MPa}$, and the change of the stress value gets slow after 3.2s. The stress form of Bar 1580 (another connection bar of tower head and tower body) is tension, contrast to bar 1581 . However, the stress change tendency of the two bars is the same, that at $0.986 \mathrm{~s}$ the stress value reaches its maximum, $39.245 \mathrm{MPa}$, and the change gets slow after $3.2 \mathrm{~s}$. 


\section{Impact Loads and Impact Coefficient}

In order to evaluate the line-broken effect of the aluminum conductor composite core on the tower, we introduce the impact coefficient $\eta[18]$ :

$$
\eta=\frac{\sigma_{\max }-\sigma_{0}}{\sigma_{0}}
$$

In the analytical formula:

$\sigma_{\max }$ - the maximum stress of the member when the conductor is broken;

$\sigma_{0}$ - - the stress value of the member under the normal operation.

Extracting the stress of the main members (Z1-Z5), the diagonal members(X1-X5), the members of the tower head (T1-T3) in the transmission tower-line system after the cable rupture (shown in chart 1), the specific locations of every members are shown in Figure 2. From table 1, we can know that the tower neck and the crank arms bear the largest impact load after the cable rupture in the transmission tower, which can reach $18.8 \mathrm{Mpa}$; The impact load acting on the middle of the transmission tower is also relatively large, reaching $3.2 \mathrm{Mpa}$; the impact load acting on the main members of the tower leg is relatively small, only up to $1.7 \mathrm{Mpa}$; the impact load acting on the diagonal members of the tower body is small as well, reaching $1.5 \mathrm{Mpa}$. We advise that we should enhance the protection for the tower neck and the crank arms in the line by using the aluminum conductor composite core, highly increase the security coefficient of the two places in the tower design and appropriately choose the steel members with higher strength as the main members of them.

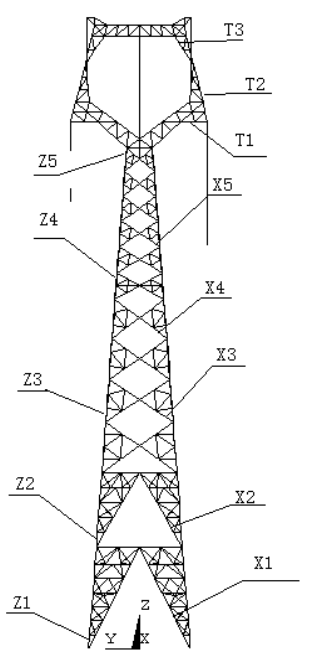

Figure 2 The figure for the specific location of the members

Table 1 The impact loads and the impact coefficients of the main members in the tower body after the cable rupture

\begin{tabular}{cccc}
\hline $\begin{array}{c}\text { Members } \\
\text { number }\end{array}$ & The stress in the gravity/MPa & $\begin{array}{c}\text { The press of } \\
\text { the side cable rupture/MPa }\end{array}$ & $\begin{array}{c}\text { Impact coefficient } \\
\eta\end{array}$ \\
\hline Z1 & -11.12 & -14.55 & 0.30861 \\
Z2 & -6.72 & -8.17 & 0.21554 \\
Z3 & -30.53 & -33.59 & 0.10029 \\
Z4 & -21.24 & -21.92 & 0.03224 \\
Z5 & -41.77 & -76.48 & 1.07902 \\
X1 & -2.14 & -5.40 & 1.52312 \\
X2 & -3.34 & -4.29 & 0.88455 \\
X3 & -7.92 & -10.39 & 0.31220 \\
X4 & -1.31 & -3.22 & 2.98661 \\
X5 & -3.28 & -4.53 & 0.38056 \\
T1 & -13.71 & -27.64 & 1.74528 \\
T2 & -20.23 & -47.19 & 1.33250 \\
T3 & -10.21 & -27.70 & 1.71344 \\
\hline
\end{tabular}

\section{Conclusion}

By simulating the cable rupture of the aluminum conductor composite core, we compare and analyze the nodes and members in the place of the tower head that bears the relatively large impact loads, and calculate the impact loads acting on the main members of the tower and the corresponding impact coefficient. Then we find that due to the cable rupture of the aluminum conductor composite core, the impact effect in the tower head and the tower neck is the largest, while the impact effect in the tower leg is small. We advise that we should enhance the protection for the tower neck and the crank arms, and rise the security coefficient of the two places in the transmission tower design.

\section{References}

1. Chen jinyan, bai junfeng. The theory and technology 
of the operation and maintenance for the transmission line[D]. Beijin: China Electric Power Press, 2009: 8890.

2. MozerJ.D, Wood William A, Hribar John A. Broken wire tests on a model transmission Line system[J]. IEEE Transactions on Power Apparatus and Systems, 1981, v100, n3, p224-228.

3. John. I.F. Broken wire analysis of transmission line systems[J]. Computers And structure, 1984, v18, n6, p1123-1126.

4. Thomas. M.B. Dynamic response of ruptured conductors in transmission lines[J]. IEEE Transactions on Power Apparatus and Systems, 1982, v101, p145-149.

5. Mozer. J. D, Pohlman J C. longitudinal load analysis of transmission line systems[J]. IEEE Transactions on Power Apparatus and Systems, 1977, v96, p78-82.

6. Ghyslaine Mcclure. Mathematical modeling of the transient response of electric Transmission lines due to conductor breakage[J]. Computers and Structures, 1987, v26, n2, p34-37.

7. Xia zhenchun, Liang zhenping, Li li. The line-broken vibration and control of the long span transmission tower-line system[J]. The journal of Wuhan University of Technology, 2008, 30(9): 84-88.

8. Bao lihua. The line-broken analysis of the transmission tower-line system[D]. Zhejiang: Zhejiang University, 2008: 18-19.

9. Tan qin, Chen hua, Wang zhonggang. The dynamic response of the transmission tower-line system under the line-broken action[J]. The Journal of Logistics Engineering Colleg, 2009, 25(3): 14-18.

10. Zhou kun, Peng jianchun. The calculation and analysis for the unbalanced and line-broken tension of the transmission line[J]. Hunan Electric Power, 2009, 29(1): 9-11.

11. Lu yuanbing. The analysis for the ice shedding and the cable rupture of the transmission line[D]. Zhejiang: Zhejiang University, 2010: 5-9.

12. Li li, Xia zhengchun, Jiang yichen, etc. The oscillation research of the transmission line breakage[J]. Engineering Mechanics, 2008, 25 (6): 165-169.

13. Shen guohui, Sun binnan, Ye yi, etc. the line-broken analysis and the line-broken tension calculation of the high voltage transmission tower[J]. the Journal of Zhenjiang University (Engineering Edition), 2011, 45 (4): 678-683.

14. Liu zhenya. the fascicle of $500 \mathrm{kv}$ transmission line of typical design for transmission and transformation engineering of the State Grid Corporation [M]. Beijin: China Electric Power Press, 2005.

15. LS-DYNA KEYWORD USER'S MANUAL[M]. California, Livermore Software Technology Corporation, 2007.

16. ANSYS/LS-DYNA instruction[Z]. 2010.

17. Shang xiaojiang, Su jianning. ANSYS/LS-DYNA dynamic analysis method and the engineering case[M]. Beijin: China Water Power Press, 2006: 274-289.

18. Bai jinze. LS-DYNA3D theoritical basis and case analysis[M]. Beijin: the Science Press, 2005: 9-11. 